\title{
Structured ring spectra and displays
}

\author{
TYLER LAWSON
}

\begin{abstract}
We combine Lurie's generalization of the Hopkins-Miller theorem with work of Zink-Lau on displays to give a functorial construction of even-periodic $E_{\infty}$ ring spectra $E$, concentrated in chromatic layers 2 and above, associated to certain $n \times n$ invertible matrices with coefficients in the Witt ring of $\pi_{0}(E)$. This is applied to examples related to Lubin-Tate and Johnson-Wilson spectra. We also give a Hopf algebroid presentation of the moduli of $p$-divisible groups of height greater than or equal to 2 .
\end{abstract}

55P42; 55N22, 55P43, 14L05

\section{Introduction}

One of the most successful methods for understanding stable homotopy theory is its connection to formal groups. By work of Quillen, a homotopy commutative and associative ring spectrum $R$ has $M U$-homology $M U_{*} R$ an algebra over the Lazard ring $L$, which carries a universal 1-dimensional formal group law, and the $M U-$ homology cooperations precisely provide $M U_{*} R$ with rules for change-of-coordinates on the formal group law.

In recent years much study has been devoted to the converse problem. Given a ring $R$ with a formal group on $R$, can we reconstruct a ring spectrum $E$ whose associated formal group lifts that on $R$ ? In addition, can more rigid multiplication (such as the structure of an $E_{\infty}$ algebra) be imposed on $E$ ? Can these constructions be made functorial?

There are several specific examples of spectra related to the chromatic filtration in stable homotopy theory for which this is of particular interest. The structure of an $E_{\infty}$ algebra on $E$ allows the construction of a symmetric monoidal model category of $E$-modules, a highly useful tool, and allows for the study of $E$-algebras. The complex bordism spectrum $M U$, which carries a universal formal group law, has an $E_{\infty}$ structure and was one of the motivating examples for the definition of such a structure. The Goerss-Hopkins-Miller theorem [4] showed the existence of canonical, functorial $E_{\infty}$ ring spectra $E_{n}$ associated to the Lubin-Tate universal deformations of height $n$ formal group laws over a perfect fields. 
However, there are numerous other spectra in the chromatic approach to stable homotopy theory, carrying formal group laws of simple type, for which $E_{\infty}$ structures are not known to exist. The most prominent example is the Brown-Peterson spectrum BP, whose ring of homotopy groups is isomorphic to a polynomial ring $\mathbb{Z}_{(p)}\left[v_{1}, v_{2}, \ldots\right]$. This spectrum carries a universal $p$-typical formal group law and serves as the basis for much computational work. Other spectra arise from the height stratification of $p$-local formal group laws. Johnson-Wilson spectra $E(n)$ have homotopy groups $\mathbb{Z}_{(p)}\left[v_{1}, \ldots, v_{n}^{ \pm 1}\right]$ covering the strata of heights 0 through $n$, and truncated BrownPeterson spectra $\mathrm{BP}\langle n\rangle$ have homotopy $\mathbb{Z}_{(p)}\left[v_{1}, \ldots, v_{n}\right]$ covering heights 0 through $n$ and $\infty$. (These definitions require making explicit choices of polynomial generators $v_{k}$ of $\mathrm{BP}_{*}$, though several formal properties of these spectra are independent of the choice.)

Of these, only certain spectra $E(1)$ and their connective covers $\mathrm{BP}\langle 1\rangle$ are classically known to admit $E_{\infty}$ structures, as they can be constructed from the periodic complex $K$-theory spectrum $K U$ and its connective cover. There exist sequences of maps

$$
\mathrm{BP} \rightarrow \mathrm{BP}\langle n\rangle \rightarrow E(n) \rightarrow E_{n}
$$

classifying progressively more restricted formal group laws, and so one might regard our problem as trying to lift $E_{\infty}$ lifts of the Lubin-Tate rings to progressively more global objects.

In 2005 Lurie announced a theorem that lifts formal groups to $E_{\infty}$ ring structures, generalizing the Goerss-Hopkins-Miller theorem. The application of this theorem requires extra data: an extension of the formal group to a $p$-divisible group. In addition, the $p$-divisible group on $R$ is required to satisfy a universality condition at each point. This specifically can be applied to produce elliptic cohomology theories and the theory of topological modular forms, and served as the basis for previous joint work with Behrens [1] generalizing topological modular forms to moduli of higher-dimensional abelian varieties that reach all chromatic levels. Joint work with Hill [5] showed that a variant of $\mathrm{BP}\langle 2\rangle$ at the prime 3 can be constructed by this method, via a $p$-divisible group on an Atkin-Lehner quotient of the Shimura curve of discriminant 14.

As the study of $p$-divisible groups originated with their connection to abelian varieties, from a geometric point of view these are some of the most natural families of 1-dimensional $p$-divisible groups. However, one of the major obstructions to understanding the associated cohomology theories is that it rests on an understanding of certain moduli of higher-dimensional abelian varieties, and in particular their global geometry. This presents barriers both because of the necessary background and because these moduli seem to be intrinsically difficult. Moreover, from the point of view of homotopy theory one does not have as many of the "designer" tools of the subject 
(see Hopkins [6]), which construct spectra with the explicit goal of capturing certain homotopy-theoretic phenomena.

The aim of this paper is to provide a method for constructing $E_{\infty}$ ring spectra from purely algebraic data. Specifically, Theorem 5.2 allows the functorial construction of even-periodic $E_{\infty}$ ring spectra with $\pi_{0}=R$ from the data of certain $n \times n$ invertible matrices with coefficients in the Witt ring of $R$. This is obtained using Zink's displays on $R$ [9], which correspond to certain $p$-divisible groups over $\operatorname{Spec}(R)$. This generalizes the Dieudonné correspondence between $p$-divisible groups over a perfect field and their associated Dieudonné modules, and in particular restricts to this structure at any perfect residue field $k$. Due to restrictions on the $p$-divisible groups constructible by these displays, the associated spectra are concentrated in chromatic height greater than or equal to 2 . In Section 5 we construct $E_{\infty}$ ring structures on spectra interpolating between Lubin-Tate and Johnson-Wilson spectra.

The layout of this paper is as follows. In Section 2 we recall the definitions of displays and nilpotent displays over a ring $R$ from [9], specifically concentrating on those in matrix form. We state the equivalence of categories between nilpotent displays and formal $p$-divisible groups on $\operatorname{Spec}(R)$ due to Zink and Lau. In Section 3 we apply Serre duality to obtain a classification of $p$-divisible groups of dimension 1 and formal height at least 2 on $R$ and give a presentation of the moduli of such $p$-divisible groups by a large Hopf algebroid. In Section 4 we study the deformation theory of nilpotent displays in matrix form over a ring $R$ and use this to give a criterion for these to satisfy the universal deformation property. Specifically, a display in matrix form determines a map to projective space $\mathbb{P}^{h-1}$ that is formally étale if and only if the associated $p$-divisible group is locally a universal deformation. In Section 5 we recall the statement of Lurie's theorem and apply it to functorially obtain even-periodic ring spectra associated to certain displays. Finally, we relate this (as in Fargues [2]) to the work of Gross and Hopkins on the rigid analytic period map [7]. In the specific case of a Lubin-Tate formal group over $R=\mathbb{W}(k) \llbracket u_{1}, \ldots, u_{h-1} \rrbracket$, there is a choice of coordinates such that the above map $\operatorname{Spec}(R) \rightarrow \mathbb{P}^{h-1}$ induces a rigid-analytic map that agrees with the Gross-Hopkins period map modulo the ideal $\left(u_{1}, \ldots, u_{h-1}\right)^{p}$.

We mention that Zink's theory of Dieudonné displays provides objects equivalent to general $p$-divisible groups over certain complete local rings. These could be applied to produce homology theories associated to universal deformations of $p$-divisible groups of dimension 1 over a field, analogous to Lubin-Tate spectra, which are worth study in their own right. However, our goal in this paper is to allow more global rather than local constructions. 
Notation For a ring $R$, we write $\mathbb{W}(R)$ for the ring of $p$-typical Witt vectors over $R$. This carries Frobenius and Verschiebung maps $f, v: \mathbb{W}(R) \rightarrow \mathbb{W}(R)$, the Teichmüller lift $[-]: R \rightarrow \mathbb{W}(R)$ and ghost maps $w_{k}: \mathbb{W}(R) \rightarrow R$. We write $I_{R}$ for the ideal of definition $v(\mathbb{W}(R))$.

Acknowledgments The author would like to thank Eike Lau, William Messing and Niko Naumann for discussions related to this material, as well as the organizers and participants of a mini-FRG on $p$-divisible groups and stable homotopy theory at UIUC in June 2009.

\section{Displays}

We will first briefly recall the classical Dieudonné correspondence. Let $k$ be a perfect field. The Dieudonné module functor $\mathbb{D}$ is a contravariant equivalence of categories between $p$-divisible groups over $\operatorname{Spec}(k)$ and finitely generated free modules $M$ over the Witt ring $\mathbb{W}(k)$ that are equipped with operators $F$ and $V$ satisfying the following properties.

- $\quad F$ is semilinear: for $x \in \mathbb{W}(k), m \in M, F(x m)=f(x) F(m)$.

- $V$ is antisemilinear: for $x \in \mathbb{W}(k), m \in M, x V(m)=V(f(x) m)$.

- $F V=V F=p$.

Classical Dieudonné theory also incorporates duality. Each $p$-divisible group $\mathbb{G}$ has a Serre dual $\mathbb{G}^{\vee}$, whose associated Dieudonné module $\mathbb{D}\left(\mathbb{G}^{\vee}\right)$ is isomorphic to the dual module

$$
\mathbb{D}(\mathbb{G})^{t}=\operatorname{Hom}_{\mathbb{W}(k)}(\mathbb{D}(\mathbb{G}), \mathbb{W}(k))
$$

equipped with Frobenius operator $V^{t}$ and Verschiebung operator $F^{t}$. The covariant Dieudonné module of $\mathbb{G}$ is the Dieudonné module of $\mathbb{G}^{\vee}$, and this provides a covariant equivalence of categories between Dieudonné modules and $p$-divisible groups.

Zink's theory of displays is a generalization of the Dieudonné correspondence. However, over a nonperfect ring $R$ defining the map $V$ is no longer sufficient. This is instead replaced by a choice of "image" of $V$, together with an inverse function $V^{-1}$.

Definition 2.1 (Zink [9, Definition 1]) A display over a ring $R$ consists of a tuple $\left(P, Q, F, V^{-1}\right)$, where $P$ is a finitely generated locally free $\mathbb{W}(R)$-module, $Q$ is a submodule of $P$ and $F: P \rightarrow P$ and $V^{-1}: Q \rightarrow P$ are Frobenius-semilinear maps. These are required to satisfy the following:

- $I_{R} P \subset Q \subset P$. 
- The map $P / I_{R} P \rightarrow P / Q$ splits.

- $\quad P$ is generated as a $\mathbb{W}(R)$-module by the image of $V^{-1}$.

- $V^{-1}(v(x) y)=x F(y)$ for all $x \in \mathbb{W}(R), y \in P$.

If $p$ is nilpotent in $R$, then $\mathbb{W}(R) \rightarrow R$ is a nilpotent thickening [9, Proposition 1.3], and $P$ being locally free on $\mathbb{W}(R)$ implies this is also true locally on $\operatorname{Spec}(R)$. In addition, $P / Q$ and $Q / I_{R} P$ are locally free $R$-modules.

Therefore, locally on such $R$ we may choose a basis $\left\{e_{i} \mid 1 \leq i \leq h\right\}$ of $P$ such that $Q=I_{R} P+\left\langle e_{d+1}, \ldots, e_{h}\right\rangle$. We refer to $h$ as the height and $d$ as the dimension of the display, and these are locally constant on $\operatorname{Spec}(R)$. As in [9], in such a basis we may define an $h \times h$ matrix $\left(b_{i j}\right)$ as follows:

$$
\begin{aligned}
F e_{j} & =\sum b_{i j} e_{i} \text { for } 1 \leq j \leq d, \\
V^{-1} e_{j} & =\sum b_{i j} e_{i} \text { for }(d+1) \leq j \leq h .
\end{aligned}
$$

These determine all values of $F$ and $V^{-1}$ :

$$
\begin{aligned}
F e_{j}=V^{-1}\left(v(1) \cdot e_{j}\right) & =\sum\left(p b_{i j}\right) e_{i} \text { for }(d+1) \leq j \leq h, \\
V^{-1}\left(v(x) \cdot e_{j}\right) & =\sum\left(x b_{i j}\right) e_{i} \text { for } 1 \leq j \leq d, x \in \mathbb{W}(R) .
\end{aligned}
$$

The image of $V^{-1}$ generates all of $P$ if and only if this matrix is invertible, or equivalently that its image under the projection $w_{0}: M_{h} \mathbb{W}(R) \rightarrow M_{h} R$ is invertible.

To aid calculation in Section 4 and further, we will refer to the inverse matrix $B=$ $\left(b_{i j}\right)^{-1}$ as a matrix form for the given display. Specifying the matrix form is equivalent to specifying the inverse matrix.

If $R$ is a perfect field of characteristic $p$, the operator $V^{-1}$ has a genuine inverse defining an antisemilinear map $V: P \rightarrow Q \subset P$, and the maps satisfy $V F=F V=p$. Under these circumstances, if

$$
B^{-1}=\left[u_{1} \mid u_{2}\right], B=\left[\frac{w_{1}}{w_{2}}\right]
$$

are block forms, then the operators reduce to the classical operators $F$ and $V$ on a Dieudonné module which is free over $\mathbb{W}(k)$ with basis $\left\{e_{i}\right\}$, and these operators have the matrix expression

$$
F\left[\frac{x}{y}\right]=\left[u_{1} \mid p u_{2}\right]\left[\frac{f x}{f y}\right], \quad V x=\left[\frac{v w_{1}}{f^{-1} w_{2}}\right]\left(f^{-1} x\right) .
$$


A map between two displays is a $\mathbb{W}(R)$-module map $P \rightarrow P^{\prime}$ preserving submodules and commuting with $F$ and $V^{-1}$. Given an isomorphism $\phi$ from $\left(P, Q, F, V^{-1}\right)$ to $\left(P^{\prime}, Q^{\prime}, F^{\prime},\left(V^{\prime}\right)^{-1}\right)$, the operators $F^{\prime}$ and $\left(V^{\prime}\right)^{-1}$ are determined uniquely by $F^{\prime}=\phi F \phi^{-1}$ and similarly for $V^{\prime}$. If these displays have matrix forms $B$ and $B^{\prime}$ respectively, we can write $\phi$ in the block form

$$
\phi=\left[\begin{array}{l|l}
a & v b \\
\hline c \mid d
\end{array}\right],
$$

and calculate that the matrix form for the display $\left(P^{\prime}, Q^{\prime}, \phi F \phi^{-1}, \phi V^{-1} \phi^{-1}\right)$ is given by the change-of-coordinates formula

$$
B^{\prime}=\left[\begin{array}{c|c}
f a & b \\
\hline p \cdot f c & f d
\end{array}\right] \cdot B \cdot\left[\begin{array}{c|c}
a & v b \\
\hline c & d
\end{array}\right]^{-1} .
$$

The associated map of displays induces the map of modules $Q / I_{R} P \rightarrow Q^{\prime} / I_{R} P^{\prime}$ given in matrix form by $w_{0}(d)$.

Given a matrix form $B$, let $\bar{B}$ be the $(h-d) \times(h-d)$ matrix in $R /(p)$ which is the image of lower-right corner of $B$ under the projection $\mathbb{W}(R) \rightarrow R /(p)$. We say that the display is nilpotent if the product

$$
f^{n} \bar{B} \cdots f^{2} \bar{B} \cdot f \bar{B} \cdot \bar{B}
$$

is zero for some $n \geq 0$. (This is independent of the choice of basis, as it is equivalent to the semilinear Frobenius map acting nilpotently on the quotient of $Q$ by $(p)+I_{R} P$.) Here $f$ is the Frobenius on $R /(p)$ applied to each entry of the matrix. A general display over $R$ is nilpotent if it is locally nilpotent in the Zariski topology.

Define a formal $\mathbb{Z}_{p}$-algebra to be a topological ring $R$ that is complete and separated in the $I$-adic topology for an ideal of definition $I$ containing $p$. We refer to a display on a formal $\mathbb{Z}_{p}$-algebra $R \cong \lim R / I^{k}$ as nilpotent if its restrictions to the $R / I^{k}$ are nilpotent.

Theorem 2.2 (Zink [9, Theorem 9]; Lau [8, Theorem 1.1]) If $R$ is a formal $\mathbb{Z}_{p}-$ algebra, there is a (covariant) equivalence of categories between nilpotent displays over $R$ and formal $p$-divisible groups on $\operatorname{Spf}(R)$.

Remark 2.3 Under this correspondence, the Lie algebra of the $p$-divisible group associated to a display $\left(P, Q, F, V^{-1}\right)$ is the locally free $R$-module $P / Q$ (see immediately following Theorem 4 of [9]). 


\section{Moduli of 1-dimensional $p$-divisible groups}

Beginning in this section we specialize to the case of topological interest: the theory of 1 -dimensional $p$-divisible groups. Unfortunately, there are very few 1 -dimensional $p-$ divisible groups to which Theorem 2.2 applies. The only ones satisfying the conditions of Lurie's theorem (see Theorem 5.1) are analogues of the Lubin-Tate formal groups.

However, the category of $p$-divisible groups has a notion of duality, compatible with a corresponding duality on the display. Serre duality is a contravariant selfequivalence of the category of $p$-divisible groups over a general base $X$ that associates to a $p$-divisible group $\mathbb{G}$ of constant height $h$ and dimension $d$ a new $p$-divisible group $\mathbb{G}^{\vee}=\operatorname{Hom}\left(\mathbb{G}, \mathbb{G}_{m}\right)$ of height $h$ and dimension $h-d$. This equivalence takes formal $p$-divisible groups to $p$-divisible groups whose Verschiebung endomorphism is topologically nilpotent. Over an algebraically closed field, this is equivalent to $\mathbb{G}$ containing no subobjects of height 1 and dimension 1 . There is a compatible notion of duality for displays $[9,1.13,1.14]$, sending a display $\left(P, Q, F, V^{-1}\right)$ to a new display $\left(P^{t}, Q^{t}, F^{t}, V^{-t}\right)$ where $P^{t}=\operatorname{Hom}(P, \mathbb{W}(R))$ and $Q^{t}$ is the submodule of maps sending $Q$ into $I_{R}$. The operators $F^{t}$ and $V^{-t}$ are determined by the formula $v\left(\left(V^{-t} f\right)\left(V^{-1} x\right)\right)=f(x)$ for $f \in Q^{t}, x \in Q$.

Composing this duality equivalence with Theorem 2.2, we find the following.

Corollary 3.1 If $R$ is a formal $\mathbb{Z}_{p}$-algebra, there is a contravariant equivalence of categories between nilpotent displays of height $h$ and dimension $(h-1)$ over $R$ and $p$-divisible groups of dimension 1 and formal height at least 2 on $\operatorname{Spec}(R)$.

Under this correspondence, the Lie algebra of the $p$-divisible group associated to a display $\left(P, Q, F, V^{-1}\right)$ is the locally free $R$-module $\operatorname{Hom}_{R}\left(Q / I_{R} P, R\right)$, and the module of invariant 1 -forms is isomorphic to $Q / I_{R} P$.

Remark 3.2 In particular, the $p$-divisible group associated to a display in matrix form $B$, with basis $e_{1} \cdots e_{h}$, has a canonical nowhere-vanishing invariant 1 -form $u$ which is the image of $e_{h}$ in $Q / I_{R} P$, and a change-of-coordinates as in Equation (1) acts on $u$ by multiplication by $w_{0}(d)$.

We can use this data to given a presentation for the moduli of $p$-divisible groups of height $\geq 2$. We recall that the Witt ring functor $\mathbb{W}$ is represented by the ring $W=\mathbb{Z}\left[a_{0}, a_{1}, a_{2}, \ldots\right]$.

Proposition 3.3 Displays in matrix form of height $h$ and dimension $(h-1)$ are represented by the ring

$$
A=\mathbb{Z}\left[\left(\beta_{n}\right)_{i j}, \operatorname{det}(\beta)^{-1}\right] \cong W^{\otimes h^{2}}\left[\operatorname{det}(\beta)^{-1}\right]
$$


The indices range over $n \in \mathbb{N}, 1 \leq i, j \leq h$. The element $\operatorname{det}(\beta)$ is the determinant of the matrix $\left(\left(\beta_{0}\right)_{i j}\right)$.

Isomorphisms between displays are represented by the ring

$$
\Gamma=A\left[\left(\phi_{n}\right)_{i j}, \operatorname{det}(\phi)^{-1}\right] \cong A \otimes W^{\otimes h^{2}}\left[\operatorname{det}(\phi)^{-1}\right] .
$$

The indices range over $n \in \mathbb{N}, 1 \leq i, j \leq h$, with the convention that $\left(\phi_{0}\right)_{i j}$ is zero if $1 \leq i \leq(h-1), j=h$. The element $\operatorname{det}(\phi)$ is the determinant of the matrix $\left(\left(\phi_{0}\right)_{i j}\right)$. The ideal $J=\left(p,\left(\beta_{0}\right)_{h h}\right)$ of $A$ is invariant. A display represented by $A \rightarrow R$ for $R$ a formal $\mathbb{Z}_{p}$-algebra is nilpotent if and only if it factors through the completion of $A$ at this ideal.

Proof The ring $W^{\otimes h^{2}}$ represents the functor

$$
R \mapsto\{h \times h \text { matrices with entries in } \mathbb{W}(R)\},
$$

and so the ring $A$ represents $h \times h$ invertible matrices $\left(\beta_{i j}\right)=B$ with entries in the Witt ring. Similarly, the ring $\Gamma$ represents pairs of a display in matrix form and an isomorphism to a second display in matrix form, according to the change-of-coordinates formula (1).

The change-of-coordinates formula, mod $I_{R}$, takes $\beta_{h h}$ to a unit times itself, and therefore the ideal $\left(p,\left(\beta_{0}\right)_{h h}\right)$ is invariant.

Suppose $R$ is a $\mathbb{Z} / p^{k}$-algebra and $A \rightarrow R$ represents a matrix $B$, which we view as the matrix form of the display. The display is nilpotent as in Section 2 if and only if

$$
\left(\beta_{0}\right)_{h h}^{p^{n}} \cdots\left(\beta_{0}\right)_{h h}^{p} \cdot\left(\beta_{0}\right)_{h h}
$$

is zero in $R /(p)$ for some $n$. This is equivalent to $\left(\beta_{0}\right)_{h h}$ being nilpotent in $R$, and so the display is then nilpotent over $R$ if and only if the map $A \rightarrow R$ factors through a continuous map $A^{\wedge} \rightarrow R$. The corresponding statement for formal $\mathbb{Z}_{p}$-algebras follows.

Corollary 3.4 The pair $(A, \Gamma)$ forms a Hopf algebroid, and the completion $\left(A^{\wedge}, \Gamma^{\wedge}\right)$ at the invariant ideal $J$ has an associated stack (in the flat topology) isomorphic to the moduli of $p$-divisible groups of height $h$, dimension 1 , and formal height at least 2 .

Proof The existence of a Hopf algebroid structure on $(A, \Gamma)$ is a formal consequence of the fact that this pair represents a functor from rings to groupoids.

Let $\mathcal{M}_{p}(h)$ be the moduli stack of $p$-divisible groups of height $h$ and dimension 1 . The universal nilpotent display on $A^{\wedge}$ gives rise to a natural transformation of functors $\operatorname{Spf}\left(A^{\wedge}\right) \rightarrow \mathcal{M}_{p}(h)$. Corollary 3.1 implies that the 2-categorical pullback 
$\operatorname{Spf}\left(A^{\wedge}\right) \times_{\mathcal{M}_{p}(h)} \operatorname{Spf}\left(A^{\wedge}\right)$, representing a pair of nilpotent displays in matrix form with an isomorphism between the associated $p$-divisible groups, is $\operatorname{Spf}\left(\Gamma^{\wedge}\right)$.

By Corollary 3.1, the resulting natural transformation of groupoid valued functors from the pair $\left(\operatorname{Spf}\left(A^{\wedge}\right), \operatorname{Spf}\left(\Gamma^{\wedge}\right)\right)$ to $\mathcal{M}_{p}(h)$ is fully faithful, and hence fully faithful on the categories of descent data for any cover. Therefore, it remains to show that the map from the associated stack is essentially surjective. Given a $p$-divisible group $\mathbb{G}$ on a formal $\mathbb{Z}_{p}$-scheme $X$ of height $h$, dimension 1 , and formal height at least 2 , there exists a Zariski open cover of $X$ by affine coordinate charts $\operatorname{Spf}\left(R_{i}\right) \rightarrow X$ small enough that each pullback of $\mathbb{G}$ to $\operatorname{Spf}\left(R_{i}\right)$ can be represented by a display in matrix form. Equivalently, there are factorizations $\operatorname{Spf}\left(R_{i}\right) \rightarrow \operatorname{Spf}\left(A^{\wedge}\right) \rightarrow \mathcal{M}_{p}(h)$. It follows that $\left(A^{\wedge}, \Gamma^{\wedge}\right)$ gives a presentation of the moduli as desired.

Remark 3.5 The Hopf algebroid described is unlikely to be the best possible. Zink's equivalence of categories shows that locally in the Zariski topology, a general $p-$ divisible group can be described in matrix form; there are more canonical matrix forms locally in the flat topology.

For example, if $h=2$ then a general matrix form

$$
\left[\begin{array}{ll}
\alpha & \beta \\
\gamma & \delta
\end{array}\right]
$$

(with $\delta$ topologically nilpotent) can be canonically reduced to the form

$$
\left[\begin{array}{cc}
0 & 1 \\
\gamma^{\prime} & \delta^{\prime}
\end{array}\right]
$$

and by adjoining elements to $R$ to obtain a solution of $f^{2} t=t \gamma^{\prime}$ we obtain a faithfully flat extension in which the matrix can canonically be reduced to the form

$$
\left[\begin{array}{cc}
0 & 1 \\
1 & \delta^{\prime \prime}
\end{array}\right] \text {. }
$$

However, it is not immediately clear whether the resulting Hopf algebroid would have any flatness properties. The existence of canonical forms at higher heights, as well as more explicit determination of the associated Hopf algebroids, merits further study.

\section{Deformation theory}

In this section we briefly study the deformations of displays in matrix form. We note that [9] has already fully interpreted the deformation theory of displays in terms of the deformations of the Hodge structure $Q / I_{R} P$. The approach there is specifically in 
terms of fixing deformations of $F$ and $V^{-1}$ and classifying possible deformations of the "Hodge structure" $Q$. For calculational reasons we will instead fix the deformation of $Q$ and study possible deformations of the operators.

Let $\mathrm{GL}_{h} \subset \mathbb{A}_{\mathbb{Z}_{p}}^{h^{2}}$ be the group scheme of $h \times h$ invertible matrices. There is a projection map $\operatorname{Spec}(A) \rightarrow \mathrm{GL}_{h}$ classifying the map that sends a display represented by a matrix $B \in \mathrm{GL}_{h}(\mathbb{W}(R))$ to the matrix $w_{0}(B)$. Let $p: \mathrm{GL}_{h} \rightarrow \mathbb{P}^{h-1}$ be the projection map sending a matrix $\left(\beta_{i j}\right)$ to the point with homogeneous coordinates $\left[\beta_{1 h}: \beta_{2 h}: \cdots: \beta_{h h}\right]$.

Let $(A, \Gamma)$ be the Hopf algebroid representing displays in matrix form, as in Proposition 3.3 .

Theorem 4.1 Let $k$ be a field of characteristic $p$, and $\operatorname{Spec}(k) \rightarrow \operatorname{Spec}(A) \subset$ $\operatorname{Spec}(W)^{h^{2}}$ be a point that defines a nilpotent display over $k$ with matrix form $B$. The composite map $\operatorname{Spec}(A) \rightarrow \mathrm{GL}_{h} \rightarrow \mathbb{P}^{h-1}$ maps the set of isomorphism classes of lifts of this display to $k[\epsilon] /\left(\epsilon^{2}\right)$ bijectively to the set of extensions of the composite $\operatorname{Spec}(k) \rightarrow \mathbb{P}^{h-1}$ to $\operatorname{Spec}\left(k[\epsilon] /\left(\epsilon^{2}\right)\right)$, ie the tangent space of $\mathbb{P}_{k}^{h-1}$ at $\operatorname{Spec}(k)$.

Proof Suppose we are given the matrix form $B$ of a display over $k$, and write in block form

$$
B=\left[\begin{array}{c|c}
\alpha & \beta \\
\hline \gamma & v \delta
\end{array}\right] \in \mathrm{GL}_{h}(\mathbb{W}(k)) .
$$

(Nilpotence of the display forces the final entry to reduce to zero in $k$.) Given any lift of the display on $k$ to a display on $k[\epsilon] / \epsilon^{2}$, Nakayama's lemma implies that any lift of the basis of the display gives a basis of the lift, whose matrix form is a lift of the matrix form over $k$.

Lifts of the matrix form $B$ to $k[\epsilon] / \epsilon^{2}$ are precisely of the form $B+s$ where $s$ is a matrix in $\mathbb{W}(\epsilon k)$, as any such element is automatically invertible. Applying the change-of-basis formula (1), we find that the lifts isomorphic to this one are of the form

$$
\left(I+\left[\begin{array}{c|c}
f a & b \\
\hline p \cdot f c & f d
\end{array}\right]\right)(B+s)\left(I+\left[\begin{array}{c|c}
a & v b \\
\hline c & d
\end{array}\right]\right)^{-1}
$$

for $a, b, c, d$ matrices in $\mathbb{W}(\epsilon k)$. The ideal $\mathbb{W}(\epsilon k)$ is square-zero and annihilated by $f$, so this reduces to

$$
B+\left(s+\left[\begin{array}{c|c}
0 & b \\
\hline 0 & 0
\end{array}\right] B-B\left[\begin{array}{l|l}
a & v b \\
\hline c & d
\end{array}\right]\right) .
$$


The space of all isomorphism classes of lifts is therefore the quotient of $M_{h}(\mathbb{W}(\epsilon k))$ by the subspace generated by elements of the form

$$
B\left(-\left[\begin{array}{c|c}
a & 0 \\
\hline c & d
\end{array}\right]-\left[\begin{array}{c|c}
0 & v b \\
\hline 0 & 0
\end{array}\right]+B^{-1}\left[\begin{array}{c|c}
b \gamma & 0 \\
\hline 0 & 0
\end{array}\right]\right) .
$$

As $B$ is invertible, this subspace consists of the $h \times h$ matrices in $\mathbb{W}(\epsilon k)$ whose final column is congruent to a multiple of the final column of $B\left(\bmod I_{R}\right)$.

However, this coincides with the kernel of the (surjective) map on relative tangent $\operatorname{spaces} \operatorname{Spec}(A) \rightarrow \mathbb{P}^{h-1}$ over $\mathbb{Z}_{p}$ at $\operatorname{Spec}(k)$.

Corollary 4.2 Suppose that we are given a display $\left(P, Q, F, V^{-1}\right)$ of height $h$ and dimension $(h-1)$ over a formal $\mathbb{Z}_{p}$-algebra $R$ in matrix form $\left(\beta_{i j}\right)$. Then $R$ gives a universal deformation of the associated 1 -dimensional $p$-divisible group at all points if and only if the map $\operatorname{Spec}(R) \rightarrow \mathbb{P}_{\mathbb{Z}_{p}}^{h-1}$ given by

$$
\left[w_{0}\left(\beta_{1 h}\right): w_{0}\left(\beta_{2 h}\right): \cdots: w_{0}\left(\beta_{h h}\right)\right]
$$

is formally étale.

Proof The ring $R$ gives a universal deformation of the associated $p$-divisible group at a residue field $\operatorname{Spec}(k)=x$ if and only if the completed local ring $R_{x}^{\wedge}$ is mapped isomorphically to the universal deformation ring of the $p$-divisible group. If $k$ is perfect, this is isomorphic to a power series ring $\mathbb{W}(k) \llbracket u_{1}, \ldots, u_{h-1} \rrbracket$. It suffices to check that the map $\operatorname{Spf}(R) \rightarrow \mathcal{M}_{p}(h)$ is formally étale at geometric points $x$. In particular, $R$ gives a universal deformation at a geometric point $x$ if and only if

- $\operatorname{Spf}(R)$ is formally smooth over $\mathbb{Z}_{p}$ at $x$, and

- the relative tangent space of $\operatorname{Spf}(R)$ over $\mathbb{Z}_{p}$ at $x$, which is the set of lifts $\operatorname{Spec}\left(k[\epsilon] / \epsilon^{2}\right) \rightarrow \operatorname{Spf}(R)$, maps isomorphically to the set of lifts of the display to $k[\epsilon] / \epsilon^{2}$.

However, because $\mathbb{P}_{\mathbb{Z}_{p}}^{h-1}$ is formally smooth over $\operatorname{Spf}\left(\mathbb{Z}_{p}\right)$, the map $\operatorname{Spf}(R) \rightarrow \mathbb{P}_{\mathbb{Z}_{p}}^{h-1}$ is formally étale at $\operatorname{Spec}(k)$ if and only if

- $\operatorname{Spf}(R)$ is formally smooth over $\mathbb{Z}_{p}$ at $x$, and

- the relative tangent space of $\operatorname{Spf}(R)$ over $\mathbb{Z}_{p}$ at $x$ maps isomorphically to the tangent space of $\mathbb{P}_{k}^{h-1}$ at $x$.

By the previous theorem, these conditions coincide. 


\section{Associated spectra}

We recall a statement of Lurie's generalization of the Goerss-Hopkins-Miller theorem (as yet unpublished) [3]. We write $\mathcal{M}_{p}(h)$ for the moduli of $p$-divisible groups of height $h$ and dimension $1, \mathcal{M}_{F G}$ for the moduli of 1-dimensional formal groups, and $\mathcal{M}_{p}(h) \rightarrow \mathcal{M}_{F G}$ for the canonical map representing completion at the identity.

Theorem 5.1 (Lurie) Let $\mathcal{X}$ be an algebraic stack, formal over $\mathbb{Z}_{p}$, equipped with a morphism

$$
\mathcal{X} \rightarrow \mathcal{M}_{p}(h)
$$

classifying a $p$-divisible group $\mathbb{G}$. Suppose that at any point $x \in \mathcal{X}$, the complete local ring of $\mathcal{X}$ at $x$ is mapped isomorphically to the universal deformation ring of the $p$-divisible group at $x$. Then the composite realization problem

$$
\mathcal{X} \rightarrow \mathcal{M}_{p}(h) \rightarrow \mathcal{M}_{F G}
$$

has a canonical solution; that is, there is a sheaf of $E_{\infty}$ even weakly periodic $E$ on the étale site of $\mathcal{X}$ with $E_{0}$ locally isomorphic to the structure sheaf and the associated formal group isomorphic to the formal group $\mathbb{G}^{\text {for }}$. The space of all solutions is connected and has a preferred basepoint.

We may then combine this result with Corollary 4.2 to produce $E_{\infty}$-ring spectra associated to schemes or stacks equipped with an appropriate cover by coordinate charts carrying displays. Rather than stating in maximal generality, we have the following immediate consequence.

Theorem 5.2 Suppose $R$ is a formal $\mathbb{Z}_{p}$-algebra and $B$ is the matrix form of a nilpotent display over $R$, with associated $p$-divisible group $\mathbb{G}$. If the associated map $\operatorname{Spf}(R) \rightarrow \mathbb{P}_{\mathbb{Z}_{p}}^{h-1}$ is formally étale, then there is an $E_{\infty}$ even-periodic $E=E(R, B)$ with $E_{0} \cong R, E_{2} \cong Q / I_{R} P$, and formal group isomorphic to the formal group $\mathbb{G}^{\text {for }}$. Given matrix forms $B, B^{\prime}$ of such displays over $R$ and $R^{\prime}$ respectively, $g: R \rightarrow R^{\prime}$ a ring map, and $\phi$ a change-of-coordinates from $g^{*} B$ to $B^{\prime}$ as in Equation (1), there exists a map of $E_{\infty}$ ring spectra $E(R, B) \rightarrow E\left(R^{\prime}, B^{\prime}\right)$ inducing $g$ on $\pi_{0}$ and lifting the associated map $\mathbb{G}^{\text {for }} \rightarrow\left(\mathbb{G}^{\prime}\right)^{\text {for }}$. This construction is functorial in $B^{\prime}$ as an object over $B$.

Proof The existence of $E(R, B)$ follows from Theorem 5.1 and Corollary 4.2. Given any such map $\operatorname{Spf}\left(R^{\prime}\right) \rightarrow \operatorname{Spf}(R)$, the maps $\operatorname{Spf}\left(R^{\prime}\right) \rightarrow \mathbb{P}^{h-1}$ and $\operatorname{Spf}(R) \rightarrow \mathbb{P}^{h-1}$ both being formally étale forces $\operatorname{Spf}\left(R^{\prime}\right)$ to be formally étale over $\operatorname{Spf}(R)$. Lurie's theorem then implies that the map lifts. 
We recall from Remark 3.2 that in matrix form there is a nowhere vanishing 1-form $u$ on the cotangent space $Q / I_{R} P$ of the formal group $\mathbb{G}^{\text {for }}$, implying that the tensor powers of the cotangent bundle are all free. This implies the strictly even-periodic structure on $E$.

Associated to a display not in matrix form, we would instead obtain a weakly evenperiodic ring spectrum whose $2 k$-th homotopy group is the $k$-th tensor power of the locally free module $Q / I_{R} P$ of invariant 1 -forms.

Example 5.3 Let $h \geq 2$ and $R=\left(\mathbb{Z}\left[u_{1}, \ldots, u_{h-1}\right]\right)_{\left(p, u_{1}\right)}^{\wedge}$. Then we have the following display over $R$ :

$$
\left[\begin{array}{cccccc}
0 & 0 & 0 & & 0 & 1 \\
1 & 0 & 0 & \cdots & 0 & {\left[u_{h-1}\right]} \\
0 & 1 & 0 & & 0 & {\left[u_{h-2}\right]} \\
& \vdots & & & & \vdots \\
0 & 0 & 0 & & 0 & {\left[u_{2}\right]} \\
0 & 0 & 0 & \cdots & 1 & {\left[u_{1}\right]}
\end{array}\right]
$$

Recall $[x]$ denotes the Teichmüller lift of the element $x$. The associated map $\operatorname{Spf}(R) \rightarrow$ $\mathbb{P}^{h-1}$ is the map

$$
\left[1: u_{h-1}: \cdots: u_{1}\right]
$$

which is the completion of a coordinate chart of $\mathbb{P}^{h-1}$ at the ideal $\left(p, u_{1}\right)$ and is therefore formally étale. Because this display is given in matrix form, there is a canonical nonvanishing invariant 1 -form $u$ and the resulting spectrum has homotopy groups

$$
\left(\mathbb{Z}\left[u_{1}, \ldots, u_{h-1}\right]\right)_{\left(p, u_{1}\right)}^{\wedge}\left[u^{ \pm 1}\right] .
$$

Here $|u|=2$. This spectrum carries an extension of a Lubin-Tate formal group to the completion of a larger closed set in affine $n$-space $\mathbb{A}^{n}$.

Example 5.4 Let $S=\mathbb{W}\left(\mathbb{F}_{p^{h}}\right)\left[u_{1}, \ldots, u_{h-1}\right]_{\left(p, u_{1}\right)}^{\wedge}$, with the same display as given in the previous example. Let $\zeta$ be a primitive $\left(p^{h}-1\right)$ 'st root of unity in $\mathbb{F}_{p^{h}}$ with Teichmüller lift $[\zeta]$. Then the group $\mathbb{F}_{p^{h}}^{\times}=\langle\zeta\rangle$ acts on $S$ with generator $\zeta$ acting by

$$
\zeta \cdot\left(u_{1}, \ldots, u_{h-1}\right)=\left(\zeta^{1-p} u_{1}, \zeta^{1-p^{2}} u_{2}, \ldots, \zeta^{1-p^{h-1}} u_{h-1}\right),
$$


and the action of $\zeta$ lifts to an action on the display via the change-of-coordinates matrix

$$
\left[\begin{array}{ccccc}
{\left[\zeta^{p^{h-1}}\right]} & 0 & & 0 & 0 \\
0 & {\left[\zeta^{p^{h-2}}\right]} & \cdots & 0 & 0 \\
& \vdots & & \vdots & \\
0 & 0 & \cdots & {\left[\zeta^{p}\right]} & 0 \\
0 & 0 & & 0 & {[\zeta]}
\end{array}\right] .
$$

This acts on the invariant 1 -form $u$ by multiplication by $\zeta$. This gives a well-defined action on the $p$-divisible group associated to the display.

Similarly, there is a Galois automorphism $\sigma$ of $S$ which acts by the Frobenius on $\mathbb{W}\left(\mathbb{F}_{p^{h}}\right)$ and acts trivially on the generators $u_{i}$. This automorphism preserves the display, and satisfies the relations $\sigma^{h}=\mathrm{id}, \sigma \zeta=\zeta^{p} \sigma$. Together these give an action of $G=\left(\mathbb{F}_{p^{h}}^{\times} \rtimes \mathrm{Gal}_{\mathbb{F}_{p^{h}} / \mathbb{F}_{p}}\right)$ on $\operatorname{Spf}(S)$ which lifts to an action on the associated $p-$ divisible group.

Theorem 5.2 implies that this lifts to an associated spectrum with an action of $G$. (More generally, there is an associated sheaf of $E_{\infty}$-ring spectra on the quotient stack $[\operatorname{Spf}(S) / / G]$.) The canonical invariant 1 -form $u$ is acted on by $\zeta$ by left multiplication, and acted on trivially by $\sigma$. The homotopy fixed point object (which is the global section object of the quotient stack) has homotopy groups

$$
\left(\mathbb{W}\left(\mathbb{F}_{p^{h}}\right)\left[u_{1}, \ldots, u_{h-1}\right]_{\left(p, u_{1}\right)}^{\wedge}\left[u^{ \pm 1}\right]\right)^{G} \cong \mathbb{Z}\left[v_{1}, \ldots, v_{h-1}, v_{h}^{ \pm 1}\right]_{\left(p, u_{1}\right)}^{\wedge} .
$$

Here $v_{i}=u^{p^{i}-1} u_{i}$ has degree $2 p^{i}-2$. This has the homotopy type of a generalized Johnson-Wilson spectrum after application of $K(2) \vee \cdots \vee K(n)$-localization.

\section{The period map}

In Section 4 we associated to each display in matrix form over $R$ a map $\operatorname{Spec}(R) \rightarrow$ $\mathbb{P}^{h-1}$. In this section we will briefly relate this, in a specific choice of coordinates, to the rigid analytic period map constructed by Gross and Hopkins [7]. The formulas of this section appear as the special case $\mathcal{O}=\mathbb{Z}_{p}$ of [2, I.2.4].

Let $k$ be a perfect field of characteristic $p$. Let $R=\mathbb{W}(k) \llbracket u_{1}, \ldots, u_{h-1} \rrbracket$, equipped with the ring homomorphism $\sigma: R \rightarrow R$ which acts by the Frobenius on $\mathbb{W}(k)$ and sends $u_{i}$ to $u_{i}^{p}$; this is a lift of the Frobenius map on $R / p$, and provides a splitting $R \rightarrow \mathbb{W}(R)$ commuting with the Frobenius. We write $J$ for the ideal $\left(u_{1}, \ldots, u_{h-1}\right)$. This ring $R$ carries the display of Equation (2). This display is a universal deformation of a 1-dimensional formal group of height $h$ over the residue field $k$, and so the 
associated $p$-divisible group on $\operatorname{Spf}(R)$ is a universal deformation of the formal group on $\operatorname{Spec}(k)$. The map $\operatorname{Spf}(R) \rightarrow \mathbb{P}^{h-1}$ of Section 4 is the map $\left[1: u_{h-1}: \cdots: u_{1}\right]$.

To translate this into the (covariant) language of the Gross-Hopkins map, we first convert the display into the dual, covariant, display, which is a free $\mathbb{W}(R)$-module $P^{t}$ with dual basis $e^{1}, \ldots, e^{h}$ and Hodge structure $Q^{t} \subset P^{t}$ generated by $e^{1}, \ldots, e^{h-1}$, and $I_{R} e^{h}$. (This Hodge structure is determined by the linear functional $\sum a_{i} e^{i} \mapsto a_{h}$.) A straightforward calculation finds that the matrix of $F^{t}$ with respect to this basis is

$$
\left[\begin{array}{cccccc}
0 & p & 0 & & 0 & 0 \\
0 & 0 & p & \cdots & 0 & 0 \\
0 & 0 & 0 & & 0 & 0 \\
& \vdots & & & & \vdots \\
0 & 0 & 0 & & p & 0 \\
0 & 0 & 0 & & 0 & 1 \\
p & p\left[u_{h-1}\right] & p\left[u_{h-2}\right] & \cdots & p\left[u_{2}\right] & {\left[u_{1}\right]}
\end{array}\right] .
$$

As in [9], there is a Dieudonné crystal associated to this display. The data of such a crystal produces: a module $M=R \otimes_{\mathbb{W}(R)} P^{t}$, a Hodge structure $Q^{t} / I_{R} P^{t} \subset M$, a $\sigma$-semilinear Frobenius map $F: M \rightarrow M$, and a $\sigma$-antisemilinear map $V: M \rightarrow M$ satisfying $F V=V F=p$. Associated to this data there is a unique connection $\nabla: M \rightarrow M \otimes \Omega_{R / \mathbb{W}(k)}$ for which $F$ and $V$ are horizontal.

We recall the construction of the period map. Let $K=\mathbb{W}(k) \otimes \mathbb{Q}$. For a sufficiently large ring $R \subset S \subset K \llbracket u_{1}, \ldots, u_{h-1} \rrbracket$ the horizontal sections of this crystal on $\operatorname{Spec}(S)$ form a vector space $V$ over $K$ of rank $h$, containing a family of rank $(h-1)-$ submodules determined by the Hodge structure. The Gross-Hopkins period map $\operatorname{Spec}(S) \rightarrow \mathbb{P}(V)$ sends a point of this rigid-analytic extension of Lubin-Tate space to the Hodge structure at that point.

Let $\Psi$ be the matrix of $F$ in this basis (the reduction $\bmod I_{R}$ of Equation (3)) and $\bar{\Psi}$ the image given by sending $u_{i}$ to 0 . There exists a deformation of the basis $\left\{e^{i}\right\}$ to a basis of horizontal sections for this connection; ie, there is a matrix $A \in \mathrm{GL}_{h}\left(K \llbracket u_{1}, \ldots, u_{h-1} \rrbracket\right)$ whose columns are annihilated by $\nabla$ satisfying $A \equiv I \bmod J$. The expression in this basis for the linear functional cutting out the Hodge structure is given by the last row of $A$.

As $F$ is horizontal, it takes horizontal sections to horizontal sections, and hence applying the Frobenius to the columns of $A$ gives a linear combination of the combinations of $A$. This implies that $\Psi A^{\sigma}=A B$ for some matrix $B$ with coefficients in $K$. Reducing mod $J$ we find that $B=\bar{\Psi}$. Thus, such a matrix must satisfy $A=\Psi A^{\sigma} \bar{\Psi}^{-1}$. (Taking a limit of iterative substitutions recovers $A$ itself.) 
As $A \equiv I \bmod J, A^{\sigma} \equiv I \bmod J^{p}$. Therefore, we find $A \equiv \Psi \bar{\Psi}^{-1} \bmod J^{p}$. Applying this to Equation (3), we find

$$
A \equiv\left[\begin{array}{ccccc}
1 & 0 & & 0 & 0 \\
0 & 1 & \cdots & 0 & 0 \\
& \vdots & & & \vdots \\
0 & 0 & & 1 & 0 \\
u_{h-1} & u_{h-2} & \cdots & u_{1} & 1
\end{array}\right] \bmod J^{p}
$$

As a result, the Gross-Hopkins map classifying the Hodge structure is congruent to $\left[u_{h-1}: \cdots: u_{1}: 1\right] \bmod J^{p}$. With an appropriate choice of coordinates we can then regard the map defined in Section 4 as an approximation of the Gross-Hopkins map.

\section{References}

[1] M Behrens, T Lawson, Topological automorphic forms, Mem. Amer. Math. Soc. 204 (2010)

[2] L Fargues, L'isomorphisme entre les tours de Lubin-Tate et de Drinfeld et applications cohomologiques, from: "L'isomorphisme entre les tours de Lubin-Tate et de Drinfeld", Progr. Math. 262, Birkhäuser, Basel (2008) 1-325 MR2441312

[3] P G Goerss, Realizing families of Landweber exact homology theories, from: "New topological contexts for Galois theory and algebraic geometry (BIRS 2008)", (A Baker, B Richter, editors), Geom. Topol. Monogr. 16, Geom. Topol. Publ., Coventry (2009) 49-78 MR2544386

[4] P G Goerss, M J Hopkins, Moduli spaces of commutative ring spectra, from: "Structured ring spectra", (A Baker, B Richter, editors), London Math. Soc. Lecture Note Ser. 315, Cambridge Univ. Press (2004) 151-200 MR2125040

[5] M Hill, T Lawson, Automorphic forms and cohomology theories on Shimura curves of small discriminant, to appear in Adv. Math.

[6] M J Hopkins, The mathematical work of Douglas C Ravenel, Homology, Homotopy Appl. 10 (2008) 1-13 MR2475614

[7] M J Hopkins, B H Gross, The rigid analytic period mapping, Lubin-Tate space, and stable homotopy theory, Bull. Amer. Math. Soc. (N.S.) 30 (1994) 76-86 MR1217353

[8] E Lau, Displays and formal p-divisible groups, Invent. Math. 171 (2008) 617-628 MR2372808

[9] T Zink, The display of a formal p-divisible group, from: "Cohomologies $p$-adiques et applications arithmétiques. I", Astérisque 278 (2002) 127-248 MR1922825 
Department of Mathematics, University of Minnesota 206 Church Street SE, Minneapolis, MN 55455

tlawson@math.umn.edu

Proposed: Paul Goerss

Seconded: Haynes Miller, Bill Dwyer
Received: 21 January 2010

Revised: 17 February 2010 